Studio

\author{
ANALITICO, SINTETICO E A PRIORI: QUESTIONI DI FORMA \\ IL DIBATTITO TRA FENOMENOLOGIA HUSSERLIANA E EMPIRISMO LOGICO \\ di Davide Bordini (bordi_82@yahoo.it)
}

[Preprint, di prossima pubblicazione su Rivista di Storia della Filosofia]

\begin{abstract}
The analytic, the synthetic and the a priori: a matter of form. The debate between Husserlian phenomenology and logical empiricism. - This article sketches out the key features of the debate on the analyticsynthetic distinction between phenomenology and logical empiricism, which took place in the early part of the twentieth century. On the one side, the author reconstructs the debate itself from an historical angle; on the other, he gives a theoretical account of the different positions and arguments. In particular, he has three main aims: a) to clarify how, according to Husserl, the analytic-synthetic opposition is to be understood as the form-matter opposition; b) to show how this position has been misunderstood by neo-empiricist philosophers, in particular by Schlick in his paper against the theory of the factual a priori; c) to point out that Husserlian theory anticipates some relevant features in the matter of analyticity (i.e. the distinction between logical analytic and extra-logical analytic truths) which will be made fully explicit only by Carnap and Quine, philosophers of the following generation.
\end{abstract}

Keywords: analyticity, analytic/synthetic distinction, Husserl, a priori, phenomenology, logical empiricism

Riassunto. L'articolo traccia le linee fondamentali del dibattito attorno all'analitico e al sintetico sorto tra fenomenologia ed empirismo logico nei primi tre decenni del XX secolo. Da un lato, l'autore fornisce una ricostruzione in senso storico del dibatto stesso, dall'altro dà una valutazione teorica delle differenti posizioni e argomenti. Gli obiettivi principali sono tre: a) chiarire come, secondo Husserl, l'opposizione analitico/sintetico sia da intendere nei termini dell'opposizione formale/materiale; b) mostrare in che modo questa posizione sia stata fraintesa dai neo-empiristi, e in particolare da Schlick nella sua critica all'a priori materiale; c) segnalare come la teoria husserliana dell'analiticità colga e anticipi aspetti rilevanti del problema dell'analiticità - quali, ad esempio, la distinzione tra verità logiche e verità per predicazione essenziale - che saranno esplicitati pienamente solo da Carnap e Quine, filosofi appartenenti alla generazione successiva.

Parole chiave: analiticità - distinzione analitico/sintetico - Husserl - a priori - fenomenologia - empirismo logico.

\title{
Introduzione
}

Non c'è dubbio che, per lungo tempo, il criterio per distinguere tra giudizi analitici e sintetici 
proposto da Kant nella Critica della ragion pura ${ }^{1}$ abbia agito alla stregua di un paradigma filosofico. Ma, come è noto, la meccanica del progresso delle idee vuole che ogni paradigma sia costantemente sottoposto al vaglio della critica e che, talvolta, venga anche messo in discussione, qualora non sia più in grado di reggere il peso degli attacchi che gli vengono rivolti.

Il paradigma kantiano è stato oggetto di diverse critiche e proposte di revisione nel corso degli anni da parte di molti filosofi. A cavallo dei primi tre decenni del XX secolo, tuttavia, esso è stato scosso nelle sue fondamenta teoriche da una contestazione radicale e profonda proveniente da due differenti scuole filosofiche: la fenomenologia husserliana e il neo-empirismo logico viennese. Tale contestazione non era stata innestata solo dalle recenti scoperte in ambito logico, matematico e fisico, ma anche (e soprattutto) dall'effetto che queste ultime avevano avuto sull'epistemologia e sul modo d'intendere i rapporti tra conoscenza, esperienza e linguaggio.

Ritornava prepotentemente al centro dell'interesse filosofico il sintetico a priori. I neo-empiristi sostenevano la completa implausibilità di quella nozione. I fenomenologi, invece, criticavano, più che la plausibilità della nozione in sé, l'oscurità del modo kantiano d'intenderla. Ciascuna di queste due prospettive, per sostenere il proprio punto di vista e basandosi sulla propria divergenza rispetto a Kant, ha introdotto un nuovo criterio di demarcazione tra l'analitico e il sintetico, riformulando a suo modo la distinzione classica.

Tutto ciò ha avuto l'effetto di originare un dibattito che va, dunque, ricostruito nelle sue linee fondamentali, affinché la legittimità delle differenti posizioni in campo possa essere soppesata attentamente e perché, alla fine, sia possibile valutare chi fornisca gli argomenti migliori. Fare tutto ciò è l'obiettivo primario di questo articolo 2 .

\section{Considerazioni preliminari}

È il caso di sottolineare fin da subito alcune caratteristiche peculiari che il dibattito in questione presenta.

Innanzitutto, esso si è trasformato, in breve tempo, in un dibattito sull'analiticità. Tutto ciò ha una ragione ben precisa. È evidente infatti che, una volta che sia stato delimitato il campo

1 Cfr. Kant 2000, pp. 39-41.

2 Il dibattito fra fenomenologi e neo-empiristi sulla distinzione analitico/sintetico e sul sintetico a priori, insieme alle questioni legate alla teoria husserliana dell'analiticità e dell'a priori materiale, sono stati oggetto di diverse ricostruzioni da parte di diversi filosofi e studiosi a noi contemporanei. Jocelyn Benoist, Roberta Lanfredini, Roberto Miraglia, Paolo Parrini, Giovanni Piana, Peter Simons, Barry Smith, Gianfranco Soldati hanno tutti scritto su questo tema, talvolta con fini più storiografici, talvolta, invece, mossi da un interesse più teorico. Il presente articolo non si schiera a favore di una di queste ricostruzioni né, quindi, si occupa di discuterle apertamente, ma aspira a porsi coerentemente nel filone di ricerca storiografica e teorica che esse tracciano. 
dell'analitico, eo ipso ne risulta delimitato anche quello del sintetico. E ciò dipende dal fatto che il modo d'intendere la questione è, fin da principio, orientato dalla convinzione (non esplicitata) secondo cui di ogni enunciato del nostro linguaggio è possibile dire se sia analitico oppure sintetico $^{3}$. Ma vi è dell'altro - e veniamo così al secondo punto che ci preme mettere in rilievo in questa sede. Definire l'ambito dell'analiticità significa caratterizzare, in qualche modo, la natura dell'a priori: significa cioè prendere posizione rispetto al fatto che la classe delle proposizioni analitiche sia (o meno) sufficientemente grande da coincidere con quella delle verità a priori. Da qui il problema del sintetico a priori e, insieme ad esso, quello concernente la natura della necessità. Infatti, assunzione comune a tutti questi filosofi è che "a priori" e "necessario" siano espressioni equivalenti.

Pertanto, all'interno di questo dibattito, prendere una certa posizione circa l'analiticità significa in realtà - come in una sorta di strana "reazione a catena" - schierarsi rispetto a tre questioni contemporaneamente, e cioè:

1. la classificazione degli enunciati in analitici e sintetici;

2. il problema dell'a priori;

3. il problema della natura della necessità.

Del resto non deve stupire che le cose stiano così: questo, infatti, è il modo in cui Kant aveva impostato il problema. Curiosamente egli, quindi, ha avuto un destino non troppo distante da quello di Isaac Newton, di cui fu grande ammiratore. Come quest'ultimo, infatti, pur essendo stato messo in discussione nel merito, è comunque rimasto un riferimento valido per quanto riguarda il metodo.

Quanto appena detto mostra come quella legata all'analiticità sia una problematica che, per come è stata posta fin dall'esordio, tende a sconfinare dalla mera classificazione logica delle proposizioni verso questioni filosoficamente piuttosto rilevanti: da quella epistemologica concernente le conoscenze a priori a quella, di stampo più metafisico, riguardante la natura delle verità necessarie ${ }^{4}$.

3 Su questo punto diverse criticità sono state sollevate da Putnam. Egli ha infatti, tra le altre cose, mostrato come questa convinzione sia uno degli elementi che contribuiscono maggiormente a creare confusione ed errori in merito al problema dell'analitico e del sintetico. La sua posizione è che vi siano degli enunciati analitici, che ve ne siano di sintetici, ma che, tra queste due polarità, si frapponga una zona di opacità fatta di proposizioni la cui classificazione in termini di opposizione analitico/sintetico o è impossibile o risulta comunque molto complessa (cfr. Putnam 1975).

4 Ora, tutto ciò potrebbe risultare sospetto e problematico a sua volta. In effetti, seguendo Kripke, si potrebbe rilevare che il problema epistemologico dell'aprioricità di talune conoscenze è altra cosa da quello metafisico riguardante le verità necessarie (cfr. Kripke 1980). Critiche come queste colgono probabilmente nel segno e aiutano a fare un deciso passo in avanti nella direzione di districare l'ingarbugliata matassa dell'analiticità. In buona sostanza, chiunque oggi voglia proporre una teoria dell'analiticità - e voglia farlo in maniera sensata - non può ignorare le ricerche di Kripke. D'altra parte l'obiettivo di questo lavoro è decisamente più modesto: qui intendo ricostruire riflessioni altrui e non presentare una mia teoria dell'analiticità. Per questa ragione, dovendo riportare i termini di un dibattito, ho ritenuto che il miglior modo per farlo fosse conservarne l'impostazione originaria, non slegando le tre questioni sopra elencate. Se ci si occupa di filosofi come Husserl, Schlick e Wittgenstein, non è possibile rendere conto fino in fondo delle loro posizioni sull'analitico e il sintetico senza esporre anche le loro teorie sull'a priori e sulla necessità. Se non si facesse così, ciò che sostengono risulterebbe davvero poco comprensibile. 
Sulla scorta di queste considerazioni dovrebbe emergere, allora, abbastanza chiaramente la ragione per cui è interessante valutare come si posizionano i filosofi citati in merito all'analiticità: essa non rappresenta per loro un banale criterio per classificare le proposizioni, ma è anche, e soprattutto, una chiave d'accesso per questioni filosoficamente piuttosto rilevanti. Una tale ampiezza tematica è, d'altra parte, la cifra più significativa sia del valore intrinseco di questo dibattito sia del valore che, conseguentemente, possono avere una sua ricostruzione e una sua valutazione.

\section{Analitico e sintetico: la riformulazione husserliana}

\subsection{Analitico e sintetico a priori}

Husserl tratta la distinzione analitico/sintetico nella Terza ricerca logica. Qui egli scrive:

possiamo definire come proposizioni analiticamente necessarie le proposizioni che hanno una verità pienamente indipendente dalla natura intrinseca delle loro oggettualità (pensate in modo determinato o in una generalità indeterminata) e dall'eventuale fattualità del caso in questione, dalla validità dell'eventuale posizione esistenziale; si tratta quindi di proposizioni che si possono "formalizzare" completamente e che possono essere comprese come casi speciali o applicazioni empiriche delle leggi analitiche o formali che sorgono validamente da tale formalizzazione. In una proposizione analitica deve essere possibile sostituire ogni materia mantenendo pienamente la forma logica della proposizione, con la forma vuota qualcosa e mettere da parte ogni posizione esistenziale passando alla forma giudicativa corrispondente, provvista di una "generalità incondizionata" ovvero del carattere di legge. ${ }^{5}$

Si tratta, dunque, di una valutazione dell'analiticità legata alla possibilità di formalizzare l'enunciato conservandone il valore di verità. Tale possibilità di formalizzazione consiste nella sostituibilità salva veritate dei termini presenti all'interno della proposizione con variabili.

Secondo questo modo d'intendere le cose sono analitiche tutte quelle proposizioni sempre vere in ragione delle loro sole componenti logiche, in cui nulla conta il significato dei termini extra-logici che vi compaiono. Possiamo, allora, enunciare la concezione husserliana dell'analiticità come segue:

(H) una proposizione è analitica se e solo se, una volta formalizzata, risulta vera per ogni valore delle sue variabili e delle sue costanti.

Esempi di proposizioni analitiche secondo Husserl saranno:

(1) non si dà il caso che qualcosa sia colorato e insieme privo di colore

5 Husserl 1968, p. 45. 
(2) qualcosa o è un suono o non lo è.

Ci troviamo, infatti, di fronte a due proposizioni interamente riconducibili a principi formali ${ }^{6}$ e, perciò, anche completamente formalizzabili salva veritate ${ }^{7}$.

Ora, se è corretto dire che tutte le proposizioni analitiche sono sempre vere, non è altrettanto giusto, secondo Husserl, sostenere che tutte le proposizioni sempre vere sono analitiche. Consideriamo il seguente set di enunciati:

(3) ogni colore ha un'estensione

(4) per un suono, non c'è altezza senza durata

(5) dati tre toni, uno è intermedio tra gli altri due

(6) l'arancione si trova sempre tra il giallo e il rosso in ordine di somiglianza ${ }^{8}$.

Anche in questo caso ci troviamo di fronte a proposizioni sempre vere. Possiamo, però, sostenere che esse siano anche analitiche? Secondo il criterio proposto in $(\mathrm{H})$, sembrerebbe di no. Infatti (3)(6) non possono essere interamente formalizzate senza che il loro valore di verità si modifichi. Proposizioni come queste, che hanno la caratteristica di essere sempre vere ma non analitiche, sono per Husserl sintetiche a priori.

Le proposizioni sintetiche a priori, non essendo leggi logiche o loro esemplificazioni, non godono della medesima generalità delle proposizioni analitiche. La loro validità è, al contrario, ben delimitata da ciò a cui si riferiscono: le verità che enunciano sono strettamente legate alle specie particolari di contenuti d'esperienza (suono, colore, estensione) che costituiscono il riferimento dell'enunciato 9 .

Il suono, il colore, l'estensione, ecc., sono ciò che Husserl chiama concetti materiali. Essi vengono da lui contrapposti ai concetti formali della logica.

\subsection{L'a priori materiale}

L'idea forte, sottesa alla riformulazione husserliana della dicotomia analitico/sintetico, è

6 (1) è un'applicazione del principio di non-contraddizione, mentre (2) è un'applicazione del principio del terzo escluso.

7 Non è difficile mostrare come (1) e (2) siano riconducibili alle seguenti scritture formali: $\left(1^{*}\right) \sim(\exists x(P(x) \wedge \sim P(x)))$, $\left(2^{*}\right) \exists x(Q(x) \vee \sim Q(x))$.

8 Prendo l'esempio (4) da Paolo Parrini (cfr. Parrini 2006, pp. 15-39), mentre gli esempi (5) e (6) sono tratti da Barry Smith (cfr. Smith, Murray 1981, pp. 47-74).

9 Non è difficile notare come (3) valga per qualsiasi colore e per qualsiasi estensione, ma non per i suoni; (4) per tutti i suoni, ma non per i colori o le estensioni; (5) per i colori e per i suoni, ma non per le estensioni; (6), infine, solo per i colori. 
sostanzialmente questa: è possibile individuare due differenti ordini di ragioni per cui un enunciato è vero a priori: la sua forma logica oppure il significato dei termini che in esso compaiono. Ricondurre una verità a priori all'una o all'altra di tali ragioni significa allora, ipso facto, determinare se essa è analitica o sintetica.

Lungo il crinale di questa differenza corre, quindi, la distinzione tra le due sfere in cui si articola l'a priori o - il che è lo stesso in questo caso - la necessità: da un lato vi sono verità a priori (o necessità) analitiche, dall'altro le verità a priori (o necessità) sintetiche.

Se le cose stanno così, allora possiamo vedere il tutto in questi termini: nella Terza ricerca logica si porta un argomento per sostenere che le verità a priori non sono tutte analitiche. La necessità, quindi, non ha natura unicamente logica. Secondo Husserl, infatti, un enunciato come:

(7) non si dà il caso che una superficie sia al medesimo tempo (tutta) rossa e (tutta) verde esprime una necessità di tipo fattuale: porta ad espressione linguistica qualcosa di extra-logico, e cioè una caratteristica strutturale del colore - segnatamente il fatto che due sfumature cromatiche diverse non possano ricoprire un medesimo punto di superficie nel medesimo tempo.

Proposizioni come (7) sono i cosiddetti a priori materiali: proposizioni che esplicitano le strutture secondo cui sono ordinati i contenuti della nostra esperienza. Asserire (7) significa, allora, esplicitare una legge essenziale valida per un'intera classe di contenuti d'esperienza - in questo caso tutti quelli riconducibili al genere colore. Leggi come questa esprimono le necessità che governano i diversi generi materiali e presiedono alla costituzione delle diverse "regioni" di oggetti. Individuarle significa costruire ontologie descrittive dei possibili contenuti d'esperienza che Husserl chiama ontologie materiali.

Sostenere l'a priori materiale, dunque, implica sostenere anche che i contenuti della nostra esperienza sono già ordinati secondo leggi insite nella loro natura e non imposte loro dalla soggettività. Pertanto, se vi è una struttura nell'esperienza - e secondo Husserl vi è -, essa va ricercata non solo nelle facoltà del soggetto, ma anche nelle sintassi obiettive secondo cui gli oggetti già si dispongono e si danno.

Proviamo a calarci brevemente sul terreno degli esempi, considerando di nuovo il caso del colore. Tramite un semplice esperimento mentale possiamo prendere una macchia di colore qualsiasi, ad esempio gialla, e provare a farla variare in continuità in direzione del rosso. Non sarà difficile anticipare che essa, prima di divenire rossa, dovrà passare per l'arancione. Ora, se seguiamo la direzione opposta di variazione, dal giallo al blu, sarà altrettanto immediato notare come la nostra macchia, prima di divenire blu, dovrà passare per il verde. Possiamo reiterare l'esperimento, 
proseguendo verso il viola e così via ${ }^{10}$.

Il punto che ci interessa sottolineare qui è il seguente: è la struttura stessa del colore a dettare tali variazioni e a rendere possibile il nostro esperimento mentale: la possibilità di anticipare le tappe del percorso della variazione. È la legalità interna al genere colore a determinare quelle "tappe forzate". Le leggi che regolano l'ontologia del colore definiscono, allora, anche il limite e l'ordine possibile della sua variazione.

La grande differenza tra la concezione kantiana e quella husserliana dell'a priori risiede proprio in questo: nell'idea che l'ordine non sia appannaggio del soggetto e che, perciò, i contenuti dell'esperienza non siano qualcosa di amorfo che deve attendere l'incontro con la soggettività per ricevere una strutturazione. Al contrario, si tratta di un insieme di contenuti organizzati secondo strutture ordinate.

La relazione tra soggetto e oggetto viene così riequilibrata nel senso di una correlazione nella quale ciascuna polarità possiede un proprio peso specifico e una certa autonomia.

La "scoperta" da parte di Husserl di una dimensione contenutistico-materiale dell'a priori - e, quindi, della necessità - è ciò che motiva l'esigenza di recuperare la distinzione analitico/sintetico e, al contempo, di riformularla tramite un criterio di demarcazione differente da quello classico kantiano, dal momento che quest'ultimo non solo non è in grado di rendere conto della differenza tra necessità formali e materiali, ma neppure la pone ${ }^{11}$.

La fenomenologia husserliana intende la nozione di sintetico a priori come a priori materiale e si pone quale compito primario l'investigazione dell'esperienza al fine di individuare e formulare le leggi che ne determinano le strutture essenziali. Il presupposto cruciale dell'indagine fenomenologica è che tali strutture sono obiettive e a disposizione, pronte per essere pienamente afferrate.

\section{L'obiezione di Schlick}

\subsection{Analitico e sintetico: la posizione del Circolo di Vienna}

È nota l'avversione dei filosofi del Circolo di Vienna nei confronti della nozione di sintetico a priori. Quest'ultima è per loro inammissibile e, in definitiva, priva di senso. Uno dei cardini teorici del neo-empirismo è, infatti, l'idea che "a priori" coincida con "analitico" e "a posteriori" con

10 Prendo questo esempio da Paolo Spinicci (cfr. Spinicci 2007, p. 156).

11 Husserl si riferisce direttamente alla teoria kantiana dell'a priori in due passaggi delle Ricerche logiche: nella Terza e nella Sesta (cfr. Husserl 1968, p. 46 e p. 504). 
"sintetico"12.

Ora, è evidente l'anti-kantismo che sta alla base di una tale concezione. Gli empiristi logici ritengono che la posizione kantiana sia sostanzialmente psicologistica: non sono i processi di pensiero a legare assieme le asserzioni che esprimono le nostre conoscenze, ma nessi logicolinguistici (sintattici e semantici) di carattere puramente formale e convenzionale. Inoltre, la concezione kantiana delle scienze, e la loro conseguente classificazione, vengono considerate obsolete e, in ultima istanza, false. In questo senso sono fondamentali sia il dibattito sulla fisica, apertosi in seguito alla rivoluzione relativistica, sia il dibattito sui fondamenti della matematica e dei suoi rapporti con la logica. La relatività einsteiniana aveva messo in crisi la fisica classica nelle sue leggi e nella sua concezione dello spazio e del tempo, mettendo perciò, contemporaneamente, sotto scacco anche l'intera epistemologia kantiana. La fisica e la geometria, infatti, si erano rivelate come discipline falsificabili, e pertanto non potevano più essere considerate a priori. D'altra parte, le ricerche di Frege, Russell, Hilbert, Wittgenstein in filosofia della matematica avevano fornito gli strumenti per la costruzione di una nuova logica interamente formale e completamente matematizzata, mostrando così la stretta affinità tra matematica e logica e intendendo la prima come una branca della seconda. A partire da qui i neo-empiristi intenderanno la matematica come una disciplina analitica e non sintetica - come invece aveva sostenuto Kant.

La distinzione analitico/sintetico, dunque, viene riformulata sulla base di una nuova classificazione delle scienze, secondo la quale vi sono da un lato scienze formali e, dall'altro, scienze reali: le prime a priori, le seconde a posteriori. Vi sono, quindi, discipline formali, che si occupano di definire i linguaggi e le loro regole, e discipline ad alto contenuto epistemologico. Le scienze naturali appartengono a quest'ultima classe. Esse hanno una portata euristica effettiva: ci parlano del mondo e ci permettono di progredire nella nostra conoscenza di esso. Tuttavia, per tale capacità esse pagano un prezzo: essere a posteriori. Una conoscenza in senso pieno, infatti, deve dirci qualcosa sul mondo, e per essere tale deve superare il vaglio dell'esperienza. Non può, dunque, darsi alcuna conoscenza riguardante oggetti d'esperienza che sia anche a priori - questa la linea di fondo di qualsiasi empirismo, e dunque anche di quello viennese.

Tuttavia, non c'è solo questo. L'impostazione kantiana, infatti, fornisce ai filosofi del Circolo di Vienna anche un'altra ragione per criticare il sintetico a priori: vediamo in che senso. L'intuizione forte di Kant consisteva nel far coincidere a priori e formale. Essa era fondata sullidea per cui una conoscenza, per potersi dire a priori, deve essere assolutamente e universalmente valida. Se le cose stanno così, allora le conoscenze a priori devono essere vere prima di e indipendentemente da

12 Questa tesi è mutuata direttamente dal Tractatus logico-philosophicus di Wittgenstein. 
qualsiasi esperienza e, perciò, devono essere anche pienamente anticipabili. Ora, che cosa è anticipabile se non l'elemento formale? La forma pare essere precisamente ciò che si può misurare nella sua validità indipendentemente da qualsiasi esperienza. Perciò l'a priori deve essere formale. A questo punto, però, si compie un ulteriore passo in avanti. Infatti, se ciò che è a priori deve essere vero in virtù della sola forma, allora è tautologico e, quindi, analitico, in quanto tautologie e proposizioni analitiche coincidono. Pertanto, anche a priori e analitico devono coincidere. Da qui, dunque, segue naturalmente l'idea che non siano possibili proposizioni contemporaneamente sintetiche e a priori. Anzi, l'ambito del sintetico viene a coincidere immediatamente con quello dell'a posteriori.

Ciò che emerge da una tale impostazione è una semplificazione del quadro sia rispetto a Kant sia rispetto a Husserl. Secondo questi ultimi, infatti, non vi è un'esatta coincidenza tra lo spazio dell'a priori e quello dell'analiticità. Da questo deriva la necessità di ammettere una classe di proposizioni molto particolari: quelle sintetiche $a$ priori.

I neo-empiristi, dal canto loro, affermano che tutto quanto è vero a priori deve essere tale in virtù della pura forma logica, il che significa per loro semplicemente questo: condizione sufficiente per stabilire la verità di una proposizione analitica è il semplice fatto di intenderne il senso. D'altra parte intendere il senso non è condizione sufficiente a stabilire la verità di una proposizione sintetica. Al contrario, è necessario "metterla alla prova": confrontarla con il mondo, con l'esperienza, e verificare se lo stato di cose che essa asserisce sussiste o meno. Potremmo anche dire così: le proposizioni sintetiche sono quelle di cui ha senso chiedersi se sono vere o false. Delle proposizioni analitiche, invece, non ha senso, in quanto esse si mostrano immediatamente come vere.

L'idea di fondo è che vi siano da un lato proposizioni sempre vere - quelle analitiche - che non dicono nulla del mondo, ma che enunciano semplicemente le regole logico-grammaticali del linguaggio tramite il quale parliamo del mondo; dall'altro, invece, abbiamo proposizioni - quelle sintetiche - che, dotate di un valore di verità e costruite sulla base delle regole grammaticali, esprimono stati di cose e dicono effettivamente qualcosa sul mondo. Da un punto di vista epistemologico queste ultime sono le più importanti, in quanto asseriscono conoscenze. Le prime godono, invece, di una priorità logica. Esempi di proposizioni analitiche saranno allora gli enunciati della matematica, o della logica, mentre quelli della fisica o della chimica saranno sintetici.

3.2 Schlick sull'a priori materiale 
A questo punto viene naturale chiedersi che tipo di proposizioni siano per i neo-empiristi viennesi quelle che, stando l'analisi di Husserl, esprimerebbero gli a priori materiali, dal momento che non possono essere sintetiche a priori.

La risposta a questa domanda ce la offre Moritz Schlick nel saggio Esiste un a priori materiale? Qui egli, muovendo un attacco rivolto in realtà all'intero impianto fenomenologico, sostiene due tesi:

1. quelle che Husserl classifica come proposizioni sintetiche a priori sono in realtà proposizioni analitiche

2. l'a priori materiale non è sostenibile.

Procediamo con ordine e vediamo nel dettaglio come si sviluppa l'argomentazione. Schlick propone come esempio paradigmatico di a priori materiale la stessa proposizione che avevamo espresso in (7) e la confronta con altri due enunciati:

(8) questa canna d'organo emette la nota la

(9) questo tessuto è rosso ${ }^{13}$.

Evidentemente vi è una differenza tra (7) e (8)-(9): esaminiamola. (8) e (9) sono proposizioni della cui verità non possiamo decidere basandoci sulla sola analisi dei significati: dobbiamo confrontarle con il mondo, dobbiamo verificare ciò che esse asseriscono. Una volta compreso il senso di (8) e (9), dunque, l'unica cosa che si può dire di esse è che entrambe possono essere egualmente vere o false. Sappiamo come sarebbe il mondo se esse fossero vere (o false). Tuttavia, decidere in un senso o nell'altro è possibile solo a posteriori, e cioè: solo dopo aver verificato se lo stato di cose che esse asseriscono sussiste effettivamente ${ }^{14}$.

$\mathrm{Al}$ contrario, per decidere della verità di (7) non abbiamo bisogno di alcuna verifica attraverso l'esperienza: basta comprendere il senso delle parole "rosso" e "verde" per poter dire che è vero, al di là di ogni ragionevole dubbio, che una medesima superficie, se è (tutta) rossa, allora non è (tutta) verde. In altre parole: è condizione sufficiente per stabilire la verità di (7) il semplice fatto che noi capiamo il significato delle parole di cui essa si compone.

(7) esprime, dunque, una verità a priori, e cioè: è una proposizione che, per esser detta vera, non necessita del confronto con l'esperienza ${ }^{15}$. Ma, se le cose stanno così, allora dovremo anche dire che

13 Schlick 1987a, p.173

14 Qui Schlick ha chiaramente in mente Wittgenstein: «comprendere una proposizione è sapere che cosa accade se essa è vera. (Dunque, una proposizione la si può comprendere senza sapere se essa sia vera)». (Wittgenstein 1964, § 4.024).

15 Dice Schlick: «nessuno nega che è unicamente e soltanto tramite l'esperienza che possiamo sapere se il vestito (di una sola tinta) portato da una certa persona in un certo momento era verde, rosso o di qualche altro colore, ma anche nessuno può negare che, una volta saputolo verde non vi è bisogno di alcuna esperienza per sapere che non è rosso»». 
(7) è analitica, dal momento che "a priori" e "analitico" si equivalgono.

A questo punto disponiamo di tutti gli elementi per esaminare la seconda tesi. Il nodo cruciale è sempre il medesimo: l'esser vera a priori di (7). Questo, infatti, determina non solo l'analiticità della proposizione, ma anche il suo carattere formale, dal momento che il presupposto di Schlick è un'equivalenza triplice tra "a priori" "analitico" e "formale". Da qui, dunque, segue naturalmente l'idea che non ci sia spazio per un a priori materiale, esattamente come non c'è spazio per un sintetico a priori ${ }^{16}$.

Sembrerebbe giocare a favore della posizione husserliana - sostiene Schlick - il fatto che i cosiddetti a priori materiali parlano di suoni, di colori: di cose che effettivamente paiono appartenere al contenuto delle nostre percezioni e della nostra esperienza, piuttosto che alla sfera formale $^{17}$. Ma la banalità che caratterizza le proposizioni come (7) deve, in qualche modo, contribuire a renderle sospette. Infatti, nonostante possa sembrare che si riferiscano a contenuti effettivi d'esperienza, in realtà esse non descrivono stati di cose possibili, ma si limitano a enunciare regole a priori: quelle regole che governano la struttura di concetti come quelli di colore o di suono e che, dunque, sovraintendono ai significati delle parole legate a tali concetti e ai loro usi possibili.

In proposizioni come (7) si rende esplicita la grammatica dei concetti, si mostrano gli usi corretti delle parole che utilizziamo, si definiscono i limiti del nostro linguaggio e si separa ciò che ha senso, da ciò che non ne ha - ciò che si può dire, da ciò che non si può dire. Pertanto, non è possibile asserire che un medesimo oggetto sia al contempo rosso e verde, e non perché nessuno ha mai avuto esperienza di un oggetto siffatto, ma perché espressioni quali "questa superficie è tanto verde quanto rossa" sono contraddizioni logiche, poiché violano le leggi che governano lo spazio logico dei concetti di colore, e perciò le leggi linguistiche dei termini di colore.

L'obiezione di Schlick si pone quindi su due piani: da un lato vi è un problema più tecnico riguardante l'analiticità di (7); dall'altro ve n'è uno più ampio che riguarda i rapporti tra le strutture della nostra esperienza, le nostre possibilità di conoscenza e il linguaggio. Di nuovo notiamo come il dibattito sull'analitico e il sintetico tenda a sconfinare verso problemi che vanno ben oltre la mera classificazione delle proposizioni.

L'idea di fondo dell'a priori materiale - e quindi della riabilitazione husserliana della nozione di sintetico a priori - era questa: le possibilità e i limiti dell'esperienza appartengono, in qualche modo, all'esperienza stessa e in essa vanno ricercati; il linguaggio porta ad espressione linguistica strutture fattuali necessarie, interne ai contenuti d'esperienza e ai loro generi. È a partire da qui,

(Schlick 1987a, p. 175).

16 Così Schlick: «le nostre proposizioni "materiali” a priori sono in verità di natura puramente concettuale, la loro validità è logica, esse hanno un carattere tautologico, formale» (ivi, p. 177).

17 Cfr. ivi, pp. 174-175. 
dunque, che l'obiezione di Schlick deve essere letta. Essa presuppone una tesi diametralmente opposta a quella di Husserl, e cioè: il linguaggio non può parlare della struttura interna degli oggetti. Tutto ciò che esso può fare è ricalcare la struttura delle relazioni fattuali esterne tra oggetti, e cioè gli stati di cose ${ }^{18}$. Proposizioni sempre vere come (7) sono in realtà pseudo-proposizioni. Esse non esprimono alcun fatto, anzi: a rigore esse non dicono nulla. Tuttavia possiedono un ruolo al limite del linguaggio: mostrare la grammatica dei termini del nostro linguaggio - in questo caso "verde" e "rosso". Ecco, dunque, il punto: (7) non ci parla dei colori, ma in essa si mostra la struttura logica dei nostri concetti di colore - in questo caso il loro essere ordinati in modo tale da non permettere che la medesima proprietà possa essere espressa da due parole diverse ${ }^{19}$. Questo è precisamente l'errore di fondo di Husserl e dei fenomenologi, secondo Schlick: confondere il piano ontologico con quello della grammatica, pensando che le regole grammaticali, che definiscono il limite di sensatezza del nostro linguaggio, siano verità sintetiche che parlano dell'esperienza e della sua struttura intrinseca.

La direzione proposta da Schlick è, dunque, opposta a quella intrapresa da Husserl. Il peso è spostato nuovamente dall'oggetto al soggetto: dalla struttura dell'esperienza alle forme delle soggettività. E, infatti, proprio questo è l'obiettivo di Schlick: negare che vi sia qualcosa come la Wesensschau - l'intuizione d'essenza; negare che sia possibile cogliere la struttura obiettiva dell'esperienza. Se una tale struttura vi è, non è affare degli esseri umani. Essa trascende loro e le loro possibilità. I limiti del linguaggio, che da un lato rendono possibile la conoscenza, impediscono dall'altro di potersi spingere così in là.

\section{Husserl e Schlick}

Chiediamoci, a questo punto, se l'obiezione di Schlick colga nel segno. È evidente che muovere un'obiezione alla teoria husserliana significa sostenere che tutte le verità a priori sono anche analitiche, il che equivale a portare delle ragioni valide che consentano di ricondurre enunciati come (7) alla classe delle proposizioni analitiche. Schlick cerca di assolvere questo compito, sostenendo che (7) deve essere analitica, in quanto è vera a priori. Ciò equivale a dire che, secondo il filosofo

18 Cfr. Schlick 1987b, pp. 47-59.

19 Come non è possibile parlare della struttura interna del mondo, così non è possibile parlare della grammatica del linguaggio, in quanto questo significherebbe già oltrepassarne i limiti. Esso, infatti, non può auto-enunciarsi. La sua grammatica, dunque, si mostra negli usi abituali e in proposizioni come (7). Così Schlick: «Sarebbe un grave fraintendimento delle nostre asserzioni se si ritenesse che le proposizioni possono parlare di strutture logiche o esprimerle nel medesimo senso in cui noi parliamo di oggetti ed esprimiamo fatti. [...] Un linguaggio non esprime ovviamente la sua grammatica, ma nell'uso del linguaggio essa mostra se stessa» (ivi, p. 58). È evidente l'influsso wittgensteiniano e, in particolare, la teoria della proposizione come immagine (cfr. Wittgenstein 1964, §§2.1-4.126). 
viennese, il criterio per l'analiticità (o tautologicità) di un enunciato è il suo esser vero a priori. È questo un buon criterio? Sembrerebbe di no. Certamente (7) è diversa, nel modo della sua verificazione, da enunciati come (8) e (9). Tuttavia, se ciò può essere sufficiente per sostenere che (7) è vera a priori, non lo è per sostenere che è anche vera per la forma - ed ecco l'errore di Schlick. Dire che (7) è vera prima di e indipendentemente da qualsiasi esperienza può bastare ad inserirla nella sfera delle verità a priori, ma non è sufficiente a ricondurla alla medesima classe di enunciati cui appartengono, ad esempio, (1) e (2) - che, lo ricordiamo, sono istanze di verità logiche. (1) e (2) sono vere a prescindere da qualsiasi riferimento all'esperienza e a prescindere dal significato dei termini che in esse compaiono. Si tratta, quindi, di enunciati realmente veri in ragione della sola forma logica. Per (7), invece, non vale lo stesso discorso: essa non può prescindere dal significato (semantica) dei termini di cui è composta. E questo è precisamente ciò che mostra Husserl, e ciò in ragione di cui egli separa l'ambito dell'analitico e del sintetico all'interno della sfera dell'a priori.

Se le cose stanno così, allora per quale ragione dovremmo considerare (7) come vera per la forma? Ora, il problema dell'argomentazione di Schlick è concentrato tutto qui: nel fatto che non fornisce una risposta convincente a questa domanda. Anzi, sostenendo continuamente che (7) è vera per la forma in quanto è vera a priori, egli non risponde affatto, ribadisce solo più volte la propria posizione. Schlick risponderebbe, se mostrasse che la forma logica di (7) è tale da renderla sempre vera; oppure portando un argomento a favore di una propria nozione di forma logica, con il quale dimostrare che il modo husserliano d'intendere tale nozione è scorretto. Ma nessuna di queste due strade viene percorsa. Non possiamo, perciò, accettare ciò che Schlick sostiene.

Vi è, però, anche un'altra ragione per rifiutare la mossa del filosofo viennese: si tratta di una ragione che riguarda il modo stesso secondo cui una buona argomentazione deve essere formulata. Schlick assume come criterio per la tautologicità di un'asserzione il suo essere vera a priori. Tale criterio è per lui una delle premesse del discorso. E questo rappresenta un errore, dal momento che non si può assumere come premessa ciò che, invece, si dovrebbe dimostrare - e cioè, in questo caso, l'equivalenza tra "tautologico" e "a priori".

Se le cose tanno così, allora la risposta di Schlick non può rappresentare per noi una reale obiezione. Essa, infatti, non funziona - e non può funzionare -, nella misura in cui non costituisce una replica puntuale rispetto a quanto Husserl mette in rilievo. Ciò che quest'ultimo ci mostra è una via percorribile per istituire una distinzione importante tra due classi di verità a priori. Il nodo centrale del suo discorso è che non vi sono ragioni sufficienti per considerare indifferenziati enunciati come (1)-(2) e come (3)-(7). La teoria appare ragionevole ed ha il pregio della chiarezza, in quanto introduce con $(\mathrm{H})$ un criterio chiaro di individuazione delle proposizioni analitiche.

Schlick avrebbe portato un'obiezione valida a tutto ciò se avesse fatto vedere, in qualche modo, 
che la classe degli enunciati veri per la forma coincide con quella delle verità a priori. Per fare questo, però, sarebbe stato necessario esplicitare un criterio per valutare l'analiticità che non coincidesse con quello per valutare se un enunciato è vero a priori. E qui è il punto. Infatti, non solo Schlick non esplicita un tale criterio, non solo non ne dispone, ma neppure lo cerca non comprendendone la necessità e dimostrando, così, di non aver afferrato la questione.

Non è, pertanto, Husserl a compiere una petizione di principio ma Schlick, non portando alcun argomento a favore della tesi secondo cui ogni verità a priori è anche formale. Di nuovo: egli assume ciò che, invece, dovrebbe dimostrare. L'errore nella strategia argomentativa è, dunque, solo la parte superficiale di un errore più profondo e più grave, e cioè: una mancata comprensione del problema che le argomentazioni husserliane intendono sollevare ${ }^{20}$.

\section{Wittgenstein}

\subsection{L'analiticità nel Tractatus logico-philosophicus}

Non è un mistero che Schlick scrivesse i suoi saggi avendo bene in mente il Tractatus logicophilosophicus di Wittgenstein: vera e propria Bibbia filosofica per tutto il Circolo di Vienna. La stessa riformulazione della distinzione analitico/sintetico operata dai filosofi viennesi presuppone le teorie del Tractatus e la sua visione della proposizione e del rapporto tra linguaggio, esperienza e

20 Se ci si interroga sulle ragioni che spingono Schlick verso la critica all'a priori materiale, allora è forse opportuno rammentare come essa derivi, in ultima istanza, dalla volontà del filosofo viennese di difendere (anche contro Husserl) uno degli assi portanti del neo-empirismo, e cioè l'idea (anti-kantiana) che abbiamo esposto secondo cui non sono possibili giudizi contemporaneamente sintetici e a priori. Ora, questa posizione, oltre ad essere motivata come abbiamo visto - dalla ricaduta epistemologica degli sviluppi del pensiero matematico scientifico, è anche supportata da un argomento che suona più o meno così. Se assumiamo che "a priori" e "necessario" sono equivalenti e che sintetiche sono quelle proposizioni che possono essere false, allora è evidente che non vi è alcuna asserzione che potrà mai essere contemporaneamente necessaria e sintetica. Perciò, abbiamo o enunciati veri $a$ priori e dunque analitici, oppure enunciati veri a posteriori sintetici. È chiaro come Schlick abbia in mente questa posizione e voglia difenderla. Ciò che egli ritiene sia da rigettare della posizione husserliana è il fatto che in essa si affermi che vi sono delle necessità riguardanti la struttura del mondo. Questa idea è inaccettabile per un empirista logico, poiché ogni configurazione di mondo è contingente. Nessuno stato di cose, nessun fatto è necessario. Per questo non possono esistere a priori fattuali. Da qui la ragione della critica schlickiana. D'altra parte, Husserl sostiene che non ogni necessità è logico-formale, poiché solo il riferimento all'esperienza e alle sue strutture permette di rendere conto, in maniera completa ed esaustiva, della verità di alcune proposizioni quali, ad esempio, "non si dà il caso che una superficie sia al medesimo tempo (tutta) rossa e (tutta) verde". Quest'ultima è, infatti, logicamente contingente. Eppure è sempre vera. Essa, dunque, deve essere necessaria in un altro senso rispetto a enunciati come " $\sim(p \wedge \sim p)$ ". La teoria dell'analiticità husserliana viene costruita a partire da qui, a partire dal fatto che si individuano due diversi sensi della necessità: l'una assoluta (o formale), l'altra condizionata (o materiale). Vi sono, dunque, due ragioni alla base dell'obiezione di Schlick: una è il tentativo di ribadire la posizione più propriamente neo-empirista sul sintetico a priori, l'altra è il fraintendimento della distinzione che Husserl traccia tra formale e materiale. Queste due ragioni, ancorché distinte, sono tuttavia strettamente connesse. È evidente, infatti, come Schlick non risponda realmente a Husserl proprio perché, fin dall'inizio, assume fra le premesse del proprio discorso la posizione tipica dell'empirismo logico secondo cui vi sono solo necessità logico-formali. Questa convinzione lo porta a fraintendere il senso della distinzione formale/materiale che egli ritiene sovrapponibile a quella analitico/sintetico. 
conoscenza.

Il caso degli enunciati che asseriscono l'esclusione dei colori viene citato nel Tractatus all'interno del più ampio capitolo delle tautologie ${ }^{21}$. Wittgenstein non ha, sostanzialmente, una posizione differente da quella di Schlick $^{22}$. Rispetto a quest'ultimo, però, il suo discorso sembra essere maggiormente perspicuo e articolato sia in merito al rapporto tra proposizioni vere per la forma, verità a priori e proposizioni analitiche, sia in merito alle conseguenze che tutto ciò ha nella considerazione degli enunciati come (7). Vediamo i passaggi in cui si articola il ragionamento wittgensteiniano.

In generale, il senso di un enunciato dipende dal suo poter essere vero o falso, e cioè: dal suo poter essere messo a confronto con i fatti. Tale confronto è possibile nella misura in cui è possibile riferire il linguaggio al mondo, proiettandolo su di esso, in virtù dell'isomorfismo strutturale che tra i due sussiste. Per sapere se un enunciato è vero o falso, dunque, dobbiamo prima sapere che cosa dice esattamente, il che vuol dire conoscere la sua struttura logica esatta. L'analisi filosofica degli enunciati ha il ruolo di portare ad evidenza tale struttura logica.

Ora, per quanto riguarda le verità a priori, si tratta di proposizioni sempre vere, e cioè: di proposizioni che non variano il proprio valore di verità, qualsiasi cosa succeda nel mondo. Ciò significa - dal punto di vista di Wittgenstein - che esse non dicono nulla. Sono indifferenti al mondo e ai fatti. Esse consegnano, dunque, al segno proposizionale tutta la loro funzione: mostrarsi come sempre vere o sempre false. Il segno proposizionale, la forma logica della proposizione, è perciò tutto quanto ci interessa.

Siamo di fronte, dunque, a qualcosa di molto particolare. Se, infatti, una proposizione ha una semantica in ragione della propria referenzialità, ciò non accade in questo caso: qui la referenzialità è ridotta a zero, così come la semantica. O meglio: qui la semantica è ricondotta interamente alla sintassi del segno proposizionale. Ma questo equivale a dire che le proposizioni vere a priori sono vere per la forma e, quindi, tautologiche. Ogni verità a priori è, dunque, una tautologia.

Se la possibilità di ricondurre una verità a priori a una tautologia non risulta evidente - ed è questo il caso di (7) - , allora il problema non sta nella proposizione, ma nel segno che la esprime. Pertanto, qualora il segno proposizionale sia tale da non rendere evidente la tautologicità di un enunciato vero a priori, ciò significa che esso va sciolto ulteriormente, tramite analisi, fino al punto in cui l'evidenza si impone. La complicazione semantica deve essere sciolta sintatticamente, fino

21 Cfr. Wittgenstein 1964, §6.3751.

22 Sul problema degli enunciati che asseriscono l'esclusione tra colori Wittgenstein e Schlick hanno avuto una conversazione a cavallo tra il 1929 e il 1930. La posizione di Schlick nel saggio sull'a priori materiale risente direttamente di quella conversazione (cfr. Waismann 1975). Qui si ritrova tra l'altro, formulato esplicitamente da Wittgenstein, l'argomento contro il sintetico a priori cui si accennava nella nota 20. Per una valutazione dell'argomento nella sua versione wittgensteiniana si veda: Simons 1992, pp. 367-371. 
scomparire del tutto, cedendo il passo alla linearità della sintassi.

(7) è, dunque, una tautologia e, quindi, una proposizione analitica. Il suo essere sempre vera dipende dalla negazione della contraddizione tra i predicati "essere rosso" ed "essere verde". Tale contraddizione, pertanto, deve essere resa evidente a livello formale nel segno proposizionale.

\subsection{Oltre il Tractatus: Some Remarks on Logical Form}

La proposta del Tractatus è piuttosto chiara. Ciononostante non è priva di problemi. In particolare va detto che, nonostante tra "verde" e "rosso" ci sia una qualche incompatibilità, non sembra tuttavia possibile ricondurre tale incompatibilità ad una contraddizione formale. Non è, infatti, sufficiente la sola semantica dei connettivi logici per concludere da " $a$ è rosso" a " $a$ non è verde" 23 , ma serve qualcosa di più, e cioè: qualcosa come una semantica di "verde" e "rosso".

Wittgenstein si rende conto di tutto ciò e nel saggio del '29, intitolato Some Remarks on Logical Form, rivede quanto aveva sostenuto nel Tractatus. In particolare ora sostiene che asserzioni contenenti termini di colore, come " $a$ è rosso" e " $a$ è verde", non si contraddicono come si contraddicono $p$ e $\sim p$. Esse, piuttosto, si escludono, e cioè: sono tali che la verità dell'una esclude immediatamente la verità dell'altra ${ }^{24}$. Le esclusioni sono dunque questo: coppie, triple..., $n$-ple di proposizioni nelle quali la verità dell'una determina la falsità di tutte le altre ${ }^{25}$.

Ad un primo sguardo, distinguendo tra contraddizioni ed esclusioni, Wittgenstein sembrerebbe andare proprio nella direzione che indicava Husserl: quella, cioè, di marcare una differenza tra proposizioni sempre vere per la forma e proposizioni sempre vere per il significato. Tuttavia Wittgenstein non percorre fino in fondo questa strada, suggerita dalla distinzione che traccia. Se lo facesse, infatti, dovrebbe concludere o che non tutte le verità analitiche sono verità formali oppure che un enunciato come (7) non è analitico. In entrambi i casi andrebbe contro la teoria del Tractatus, secondo cui ogni verità a priori è una tautologia e, quindi, è anche una verità analitica. Questa idea è, invece, proprio ciò che Wittgenstein vuole difendere strenuamente, ancora nei Remarks. Per questo egli sceglie una terza via: sostenere che (7) è tautologica. Questa, però, è una soluzione non percorribile - o almeno non è percorribile così come la presenta Wittgenstein nel '29. Egli infatti dovrebbe motivare in che senso le esclusioni sono tautologie, e cioè verità logiche, a fronte del fatto che ha appena sostenuto che non sembra possibile determinare il loro valore di

23 Cfr. Ramsey, Moore 1927, p. 167.

24 Wittgenstein 1929, pp. 168-171.

25 Questa distinzione è interessante innanzitutto perché implica un ripensamento della teoria dell'atomismo logico del Tractatus. In questa sede, però, non ci occuperemo di questo. Rimando, quindi, a: Proops 2009. 
verità unicamente tramite la semantica dei connettivi logici ${ }^{26}$.

Una via praticabile, per ovviare a questo problema, sarebbe sostenere che le regole della logica non sono solo quelle che determinano la semantica dei connettivi, ma tutte le regole di un linguaggio, anche quelle che governano il significato di parole come "rosso" e "verde"27. Tuttavia questa posizione sarebbe problematica, dal punto di vista di Wittgenstein, in un altro senso: implicherebbe, infatti, far cadere la distinzione appena tracciata tra esclusioni e contraddizioni che sembrava fondarsi proprio sulla volontà di mettere in luce una differenza tra il significato dei connettivi logici e il significato delle altre parole linguaggio.

Anche ciò che sostiene Wittgenstein presenta, dunque, non poche ambiguità e oscurità. Il punto di forza della teoria del Tractatus risiedeva nel fatto che essa definiva dei criteri chiari per tautologicità e analiticità. Dal Tractatus emergeva questo: un enunciato è analitico se e solo se è tautologico, ed è tautologico se e solo se è vero in ragione delle sue sole componenti formali - dove "componenti formali" era equivalente a "componenti logiche". Se, però, non si trova un modo convincente per ricondurre l'incompatibilità fra colori ad una contraddizione formale - e la distinzione tra esclusioni e contraddizioni non sembra essere molto convincente - , allora tutto il discorso diventa più opaco e ci si trova sostanzialmente nelle stesse condizioni in cui ci si trovava di fronte all'obiezione di Schlick: non è chiaro perché (7) dovrebbe contare come analitica, dal momento che non è chiaro perché essa sia una tautologia.

Gli enunciati che asseriscono incompatibilità fra colori sembrano mettere in crisi in qualche modo la teoria del Tractatus, ripresa anche da Schlick, secondo cui la struttura logica dei fatti è (e deve essere) riprodotta esattamente dalla forma logica della proposizione ed è pienamente ricavabile a partire da quest'ultima. Nel caso di asserzioni come (7), infatti, emerge come l'analisi logica di una proposizione non sia sempre uno strumento sufficiente per cogliere pienamente la struttura dei fatti. Di ciò, in qualche senso, Wittgenstein si rende conto ${ }^{28}$, diversamente da Schlick.

Ora, però, se Husserl da un lato ha ben presente la differenza tra la forma degli enunciati e la

26 Wittgenstein ritiene che sia sufficiente costruire una tavola di verità per le esclusioni (diversa da quella della congiunzione) perché si possa dire che esse sono vere per la forma (cfr. Wittgenstein 1929, p. 170). Tuttavia, questa assomiglia molto ad una mossa $a d$ hoc. Infatti, se l'incompatibilità tra " $a$ è rosso" e " $a$ è verde" è formale, allora questo deve essere evidente sulla base della forma, e cioè della sola semantica dei connetivi logici. Se, invece, noi basiamo l'incompatibilità tra quei due enunciati su un'analisi semantica dei termini che in essi compaiono - analisi che ci porta alla costruzione di una tavola di verità particolare - e usiamo questo procedimento per sostenere che si tratta di una specie differente di incompatibilità formale, allora la nostra argomentazione è sospetta. In particolare sembra che usiamo la parola "formale" in due modi differenti: in un senso, quando parliamo delle contraddizioni e in un altro senso, diverso dal primo, quando parliamo delle esclusioni. E questo è ancor più sospetto, dal momento che insinua il dubbio di un uso ambiguo del termine "forma" da parte di Wittgenstein.

27 È questa la strada che seguirà Carnap.

28 Nei Remarks è lo stesso Wittgenstein a dire: «we can only arrive at a correct analysis by what might be called the logical investigation of the phenomena themselves, i.e., in a certain sense a posteriori, and not by conjecturing about a priori possibilities» (Wittgenstein 1929, p. 163). 
forma dei fenomeni e la pone chiaramente, Wittgenstein dall'altro sembra inizialmente cogliere questa distinzione per metterla però poi subito da parte. Sostenere infatti coerentemente quella distinzione equivarrebbe ad ammettere che l'isomorfismo linguaggio-mondo non sia un modello adeguato. E questo per lui è rischioso: nei fatti, dal suo punto di vista, significherebbe aprire la porte all'idea secondo cui è possibile un accesso ai fenomeni indipendente dal linguaggio. Ma questa posizione - che è, poi, quella husserliana - è l'esatto contrario rispetto a quella che Wittgenstein vuole qui difendere.

Nei Remarks, in sostanza, si lavora ancora all'interno del framework del Tractatus, e cioè con un apparato sostanzialmente insufficiente a rendere conto del problema del rapporto complesso che c'è tra forma della proposizione e contenuto della proposizione - dal momento che nel Tractatus si esclude fin da principio che sia possibile uno scarto tra queste due polarità ${ }^{29}$. Tutto ciò origina oscurità e ambiguità.

\section{Husserl, Schlick e Wittgenstein: bilancio di un confronto}

Tracciamo a questo punto un bilancio. Più sopra notavamo come l'obiezione di Schlick si ponesse su due piani: uno più ristretto (e più tecnico), legato alla valutazione dell'analiticità di una classe di enunciati; uno più ampio, che coinvolge il rapporto tra linguaggio, suo riferimento al mondo e le nostre possibilità di conoscenza. Non è difficile vedere come questa duplicità di piani appartenga all'intero dibattito che abbiamo ricostruito. Pertanto, le valutazioni che di esso faremo saranno scandite secondo questo doppio binario: partiremo dalla questione più tecnica per esaminare, poi, le questioni filosofiche di fondo che si celano al di sotto di essa.

Per quanto riguarda il primo punto, innanzitutto, vale la pena di sottolineare quanto segue. Vi è qualcosa su cui Husserl, Schlick e Wittgenstein non sembrano essere molto distanti: secondo tutti e tre, infatti, le proposizioni analitiche sono tali perché completamente formali/formalizzabili ${ }^{30}$. La differenza risiede, piuttosto nel dire quali siano gli enunciati completamente formali/formalizzabili.

Ora, gli argomenti che Schlick e Wittgenstein forniscono non sembrano scalfire la posizione husserliana, per due ragioni. In primo luogo le loro sono, con sfumature diverse, due petizioni di principio. In secondo luogo, e in maniera conseguente, non si affronta il punto che Husserl pone in

29 Wittgenstein raffinerà la propria analisi e giungerà alla teoria dei giochi linguistici. I Remarks dunque rappresentano il primo passaggio di un percorso più ampio di ripensamento, che porterà alla fine Wittgenstein ad abbandonare definitivamente la teoria del significato sostenuta nel Tractatus.

30 Tra l'altro, in anni successivi, Husserl mostra esplicitamente di conoscere e accettare la nozione wittgensteiniana di tautologia e di considerarla come un utile completamento alle sue ricerche sull'analiticità (cfr. Husserl 1966, pp. 412-415). 
maniera chiara, e cioè che vi è una differenza tra le componenti logico-formali del linguaggio e le sue componenti extra-logiche. In definitiva, sia Schlick sia Wittgenstein si pongono ancora al di qua rispetto a tutto ciò.

Stando così le cose, tra le tre la proposta husserliana appare, dunque, come quella più convincente e più ragionevole: non abbiamo ragioni sufficienti per metterla in discussione e, quindi, per sostenere che l'analitico debba necessariamente coincidere con l'a priori.

Possiamo, quindi, decretare la vittoria di Husserl? Forse ancora no, purché si avanzi una nuova definizione di "analiticità", che abbia almeno due caratteristiche: a) deve tenere conto del fatto che proposizioni come (7), pur essendo verità a priori, non sono tautologie; b) deve fare tutto questo rimanendo all'interno di una prospettiva neo-empirista, che neghi la possibilità di un sintetico $a$ priori. Una definizione con tali requisiti è la seguente:

(C) un enunciato è analitico se e solo se è tautologico o è tale che sia sufficiente intendere il suo senso per stabilirne la verità.

(C) sottointende l'idea di analiticità che ha elaborato Carnap ${ }^{31}$. In base ad essa dividiamo le proposizioni in due grandi classi: le proposizioni analitiche da un lato, che coincidono con le verità a priori; dall'altro le proposizioni sintetiche, che coincidono con l'a posteriori. All'interno della classe delle proposizioni analitiche, poi, troviamo le due sottoclassi di verità a priori che abbiamo distinto sopra: quelle logiche e quelle extra-logiche.

Il punto centrale della nuova definizione è che essa considera le tautologie come una sottoclasse all'interno dell'insieme più ampio della proposizioni analitiche, non limitando a tale sottoclasse l'intero ambito dell'analiticità. Abbiamo, dunque, una definizione un po' più larga, motivata dall'idea secondo cui tutte le verità logiche (tautologie) sono anche analitiche, ma non tutte le verità analitiche sono verità logiche (tautologie) - idea che aveva trovato conferma anche nelle nostre considerazioni.

Abbiamo qualche ragione per considerare (C) come qualcosa che si avvicina molto a una definizione standard di "analitico". Oggi, infatti, la maggior parte dei filosofi si basa proprio su questa concezione dell'analiticità. Del resto è significativo che lo stesso Quine utilizzi come riferimento per il suo celebre attacco all'analiticità in quanto tale proprio l'idea sottointesa da (C). Evidentemente egli la ritiene quella più esemplificativa - se non quella standard. D'altronde il

31 Si tratta della nozione di analiticità che Carnap va elaborando a partire dagli anni ' 40 (cfr. Carnap 1956, Awodey 2007, Coffa 1987, Quine 1961, Quine 1966). In effetti, egli non distingue esplicitamente tra verità logiche ed extralogiche, ma si limita ad elaborare un approccio semantico alle verità analitiche, che implicitamente ammette l'insufficienza di un resoconto in termini puramente sintattici. Il primo ad esplicitare la differenza tra le due tipologie di proposizioni analitiche fu, per una strana ironia della sorte, proprio colui che è conosciuto per aver sottoposto ad un duro attacco l'analiticità: Willard Van Orman Quine (cfr. Quine 1961, pp. 22-23). 
bersaglio di una critica deve essere la versione standard di una teoria.

A questo punto la domanda è: (C) può mettere in crisi la teoria di Husserl? Risponderemo in due modi.

La prima risposta è decisamente negativa. La differenza tra la definizione standard e quella husserliana, infatti, sembra essere solo terminologica. Ciò che preme a Husserl è mettere in rilievo la differenza tra proposizioni vere in virtù del significato e proposizioni vere per la forma. E su questo $(\mathrm{C})$ e $(\mathrm{H})$ concordano: tra le due non c'è contraddizione, tant'è che è possibile passare dall'una all'altra aggiungendo, o togliendo, una clausola - volendo $(\mathrm{H})$ potrebbe addirittura essere una parte di (C). Resta evidentemente una differenza: la prima non limita la classe dell'analiticità alle proposizioni tautologiche, mentre la seconda lo fa. Ma non sembra questa una differenza sostanziale: una volta che abbiamo posto le tautologie e le proposizioni vere per il significato come due classi indipendenti di proposizioni, il tutto ruota attorno a quale criterio preferiamo usare per la nostra classificazione. La scelta tra una definizione più larga e una più stretta, dunque, sarà motivata da questioni esterne ai criteri stessi, che giustifichino un uso del termine "analitico" più o meno ampio.

La seconda risposta è altrettanto negativa, ma sposta le nostre considerazioni dal piano tecnico del discorso a quello più filosofico. Abbiamo visto come l'opposizione tra $(\mathrm{C})$ e $(\mathrm{H})$ non risulti inconciliabile in senso assoluto, ma solo in quanto espressione di differenti visioni filosofiche contrapposte. Ciò significa che la vera partita si gioca altrove rispetto alla distinzione analitico/sintetico. L'oggetto del contendere qui, infatti, non è più una classificazione delle proposizioni in analitiche e sintetiche, ma diventa la nozione di sintetico a priori in quanto tale, e cioè: se l'a priori possa essere "materiale", oppure se esso sia una questione prettamente linguistica; se l'esperienza possieda strutture sue proprie oppure se essa sia modellata sulle strutture linguistiche; se sia possibile un accesso diretto al mondo oppure se tale accesso sia irrimediabilmente mediato dal fatto di avere un linguaggio. Tutto ciò ha poco a che vedere con la nozione di analiticità. Per quanto le due questioni si tocchino, altro è dibattere su quali proposizioni esprimono verità necessarie, altro è chiedersi se vi siano conoscenze valide prima di e indipendentemente da qualsiasi esperienza - ed è quest'ultimo il punto su cui si scontrano empirismo viennese e fenomenologia.

Ora, (C) non rappresenta di per sé un'obiezione né al sintetico a priori né alla teoria dell'a priori materiale. Essa, semplicemente, fornisce una definizione di "analiticità" che ci permette di evitare il sintetico a priori, qualora lo ritenessimo già inutile, sospetto o insensato. (C) serve solo a supportare un'opzione filosofica particolare che deve già essere stata scelta: non rappresenta certo la ragione dirimente in base alla quale scegliere l'opzione neo-empirista ai danni di quella 
fenomenologica. Essa mostra che vi è una teoria dell'analiticità diversa da quella di Husserl, ma non mostra che quest'ultima è falsa.

A conclusione del nostro bilancio, quindi, possiamo dire quanto segue. Le argomentazioni husserliane intorno all'analiticità sembrano essere quelle che colgono più a fondo la dimensione del problema, e le sue implicazioni, rispetto a quelle di Schlick e Wittgenstein. Husserl infatti non solo intravede, ma pone anche chiaramente (e coerentemente) la distinzione tra proposizioni sempre vere per la loro forma logica e proposizioni sempre vere per il significato. A partire da qui egli imposta la propria teoria dell'analiticità; gli altri due o non vedono questa distinzione (Schlick), oppure la intuiscono in maniera solo parziale e, comunque, non la seguono fino in fondo (Wittgenstein). Pertanto, anche le obiezioni e le argomentazioni che essi portano risultano fuori bersaglio, in quanto mancano proprio quel punto su cui, invece, Husserl si concentra maggiormente.

Proprio qui, dunque, risiede un altro grande punto d'interesse che ci sottopone il dibattito tra fenomenologi e neo-empiristi sull'analitico e il sintetico, e cioè: l'emergere di una nozione di analitico come quella husserliana, assolutamente non banale per un filosofo dell'inizio del XX secolo, in quanto pone in maniera chiara e forte la distinzione tra componenti logiche ed extralogiche di un linguaggio, mostrando in questo modo di anticipare e cogliere aspetti di un dibattito che sarà sviluppato pienamente da filosofi come Carnap e Quine, appartenenti a una generazione successiva.

\section{La nozione di analitico in Husserl: analiticità linguistica e ontologica}

È evidente che tutti i filosofi di cui abbiamo parlato profondono non pochi sforzi nella ricerca di una definizione soddisfacente di "analiticità". Tuttavia, il motivo per cui lo fanno è un'urgenza legata più a interessi "interni" allo sviluppo delle loro ricerche, che non all'incalzare di un dibattito "esterno". O meglio: è evidente che i risultati cui pervengono sono frutto di un dibattito - un dibattito che, però, non è quello tra fenomenologia ed empirismo. Quest'ultimo vede scontrarsi teorie dell'analiticità già elaborate precedentemente e indipendentemente. Carnap, Husserl, Schlick e Wittgenstein conducono le proprie ricerche sull'analiticità e poi le riversano nel dibattito sull'a priori.

Se le cose stanno così, allora è evidente come le due differenti nozioni di analitico sottointendano anche due progetti filosofici radicalmente differenti. E se è vero che le teorie dell'analiticità di questi filosofi sono state elaborate in maniera parallela e poi riversate in un dibattito, allora il rischio che ci siano degli spazi di incomprensione è decisamente elevato. Ed in effetti così è stato. Ciò che interessa Carnap, Wittgenstein o Schlick è soprattutto l'analisi 
linguistica degli enunciati. Pertanto anche la loro teoria dell'analiticità sarà legata ad una prospettiva linguistica. Husserl è interessato, invece, a un'indagine fondativa di carattere ontologico rispetto all'esperienza e ai suoi contenuti, e la sua teoria dell'analiticità si pone coerentemente su questo livello.

Da ultimo, mi propongo, quindi, di tracciare brevemente il profilo di questa differenza. In tal modo dovrebbero emergere anche alcune ambiguità di fondo - non esplicitate e forse nemmeno colte dai protagonisti - che attraversano tutto il dibattito e che stanno anche alla base di alcune reciproche incomprensioni e fraintendimenti.

Tutto ruota attorno al diverso modo d'intendere la nozione di forma logica e, in definitiva, la logica stessa. Vediamo in che senso.

Consideriamo questo enunciato:

(10) ogni intero ha delle parti.

La sua forma logica sarà:

$$
\left(10^{*}\right) \forall x \exists y(P(x) \rightarrow R(y x))
$$

Ciò che risulta immediatamente evidente è che $\left(10^{*}\right)$ non è una verità logica, e cioè che non è una tautologia. Possiamo immaginare delle sostituzioni per il predicato " $P$ " e per la relazione " $R$ " tali che $(10 *)$ risulti falsa. Tuttavia, (10) è sempre vera: non c'è intero che sia privo di parti. È, dunque, naturale concludere che (10) è analitica per la definizione standard, ma non lo è secondo la definizione husserliana. In realtà le cose non stanno esattamente così: è, infatti, lo stesso Husserl a proporre (10) tra gli esempi di proposizioni analitiche ${ }^{32}$. A questo punto emerge il nodo della forma logica.

Vi è un senso in cui ricondurre un enunciato alla sua forma logica significa ricondurlo alla sua forma proposizionale. In questo caso si fa uso di una nozione puramente linguistica di forma logica. Tale nozione è propria di filosofi come Carnap, Schlick e Wittgenstein. Per questi filosofi la logica si occupa innanzitutto di strutture linguistiche, che solo in un secondo momento possono essere riferite o proiettate sul mondo. Da questo modo d'intendere la logica e la forma logica discende una nozione di analiticità che chiameremo linguistica. La definizione standard definisce questa analiticità $\mathrm{e}$, in quest'ottica, distingue tra verità analitiche logiche ed extra-logiche e, successivamente, tra queste ultime e tutte le altre proposizioni.

Secondo Husserl, invece, la logica non ha un carattere puramente convenzionale-normativo, ma è referenziale. L'analisi del linguaggio non si ferma al linguaggio stesso, ma va oltre. In definitiva, 
le proposizioni sono analitiche o sintetiche a seconda che parlino di oggetti, proprietà o relazioni interne alla sfera del formale oppure a quella materiale. Dunque, la ragione ultima dell'analiticità o della sinteticità di un enunciato rimanda oltre la dimensione linguistica e chiama in causa la natura dei rapporti che sussistono tra oggetti formali e tra oggetti materiali.

Gli oggetti formali sono variazioni sul "qualcosa in generale". Il "qualcosa in generale" è l'ambito dell'oggetto in quanto tale, e cioè dell'oggetto privo di qualsiasi determinazione e inteso come mero correlato intenzionale di un atto intenzionale qualsiasi ${ }^{33}$.

Relazioni, numeri, insiemi, interi, parti - e in generale tutti gli oggetti logico-matematici - sono oggetti formali ${ }^{34}$, in quanto ottenuti e ottenibili sulla base di operazioni di costruzione all'interno del dominio dell'oggetto in generale ${ }^{35}$. Ora, ciascun oggetto sarà una variazione specifica individuata da regole di costruzione specifiche, espresse tramite assiomi. In base a tali assiomi saranno poi derivabili interamente tutte le proprietà di quella specie di oggetto formale. Ogni oggetto formale è dunque il correlato di una teoria assiomatica ${ }^{36}$.

Per questo, allora, le proposizioni che parlano degli oggetti formali (e delle loro proprietà) sono analitiche: perché o sono assiomi della teoria oppure sono suoi teoremi, e cioè: o sono le regole stesse in base a cui quella specifica varietà di oggetti è costruita oppure seguono per conseguenza logica da quelle regole.

Se queste sono le premesse, allora individuare la forma logica di un enunciato vuol dire individuare la forma dei rapporti sussistenti tra i suoi referenti oggettuali. (10), dunque, sarà la forma logica di un enunciato come:

(11) ogni casa ha un tetto.

33 È evidente che, per giungere a tale nozione, operiamo quella che Husserl chiama "riflessione" mettendo a tema non una particolare specie di atti intenzionali, ma l'intenzionalità stessa nella sua struttura essenziale, la quale è tale da prevedere che per ogni atto è dato anche un contenuto, e cioè: qualcosa a cui quell'atto si riferisce. Dice a tale proposito Husserl: «il concetto di qualcosa deve manifestamente la sua origine alla riflessione sull'atto psichico del rappresentare, al quale è dato come contenuto precisamente ciascun oggetto determinato» (Husserl 2001, p. 122).

34 Cfr. Husserl 1968, p. 42.

35 Qui emerge un altro problema interessante: quello di ciò che conta come formale e, quindi, come termine logico. Come scrive Tarski: «the division of terms into logical and extra-logical also plays an essential part in clarifying the concept "analytical"» (Tarski 1956, p. 419). È davvero complesso tracciare un confine definitivo tra ciò che è logico e ciò che extra-logico. Tuttavia, oggi si tende a considerare come parti logiche di un enunciato i connettivi, i quantificatori ed eventuali operatori come quelli modali - e cioè quelle parti più spiccatamente sintattiche di un linguaggio. Del resto si tratta di una distinzione non arbitraria, ma neppure fondata su basi assolutamente oggettive. Per citare di nuovo Tarski: «no objective grounds are known to permit us to draw a sharp boundary between the two groups of terms». (Tarski 1956, p. 418). Per quanto riguarda Husserl, invece, possiamo dire che non la pensa esattamente così. Egli, infatti, considera intero e parte come concetti formali, appartenenti alla classe delle categorie oggettuali, e quindi come oggetti logici a pieno titolo. Non possiamo qui entrare nel merito di questo problema , rimandiamo quindi a: Hacking 1979, Sher 1991. Per i nostri scopi è sufficiente questo: la ridefinizione di Husserl di ciò che è analitico è strettamente legata ad una ridefinizione dello spazio del logico. Tutto ciò comporta naturalmente una presa di posizione rispetto a ciò che si considera come logico e ciò che si ritene extra-logico. Su questo si veda anche: Miraglia 2006, pp. 108-111.

36 Cfr. Husserl 2002a, pp. 173-174. 
Ciò equivale a dire che (10) esprime la regola formale che presiede al particolare rapporto sussistente tra gli oggetti di cui parla (11): la regola in virtù della quale è possibile stabilire a priori che è sempre vero che ogni casa ha un tetto, delle mura, ecc. In altre parole: (10) è la ragione formale della verità di proposizioni come (11).

È, dunque, il riferimento della proposizione a rapporti tra oggetti formali a determinare l'analiticità della proposizione stessa e di conseguenza il suo essere sempre vera. (10) è analitica nello stesso senso in cui è formale, e cioè in quanto esprime rapporti tra oggetti formali ${ }^{37}$.

Da questa concezione della forma $\operatorname{logica}^{38}$ ne deriva una dell'analiticità che chiameremo ontologica, più larga rispetto a quella linguistica, in quanto la comprende e la oltrepassa. Tale ampiezza corrisponde all'ampiezza che, per Husserl, è propria della sfera del formale, all'interno della quale si intrecciano $\mathrm{i}$ due sensi (linguistico e ontologico) dell'analiticità che abbiamo esplicitato. Nelle pagine husserliane un tale intreccio è costante e non sempre facilmente districabile. Del resto, Husserl non ha alcuna necessità urgente di separare un piano dall'altro ${ }^{39}$. Il suo discorso risulta perfettamente coerente. Tuttavia, non appena ci rivolgiamo per un confronto nella direzione di una prospettiva teorica differente, i problemi e le difficoltà possono sorgere e, insieme ad essi, le incomprensioni. In particolare, viene alla luce una certa ambiguità nell'uso del termine "analitico", che è data dalla sovrapposizione dei due sensi che abbiamo distinto e che rende piuttosto complesso riordinare le cose e portare avanti il confronto.

Giunti a questo punto, allora, dovremo dire che quanto emerge dal dibattito tra fenomenologi e

37 Se il ruolo del rifermento è decisivo, perché Husserl sembra trattare nozioni come quella di intero e parte come variabili? In effetti, queste due nozioni non sono esattamente delle variabili. Esse, infatti, sono indeterminate rispetto ai contenuti materiali, ma sono determinate rispetto agli altri oggetti formali. Da qui segue che non è possibile operare sostituzioni indiscriminate di "intero" e "parte" negli enunciati in cui questi termini compaiono. Essi sono rimpiazzabili con qualsivoglia genere di contenuti, a patto che tali contenuti siano reciprocamente legati da una struttura intero-parte. Per poter sostituire opportunamente in (10), dobbiamo già avere presente la teoria dell'intero e delle parti e andare a recuperare esempi adeguati.

38 La nozione husserliana di forma logica deriva da quella di Bolzano. Cfr. Bolzano 1973, Benoist 1997, Miraglia 2006, Simons 1992.

39 È necessaria una precisazione importante. L'intreccio di analiticità linguistica e ontologica in Husserl non è esattamente frutto di una confusione, ma piuttosto è l'esito naturale della concezione stessa che Husserl ha dell'analiticità e, prima ancora, del formale. Se il versante linguistico e quello ontologico sono così tanto intrecciati, allora ciò accade perché è la natura stessa degli oggetti formali ad essere duplice: infatti essi sono da un lato oggetti, dall'altro categorie. Noi possiamo, perciò, tracciare una linea di confine tra i rapporti analitici tra oggetti e quelli tra espressioni linguistiche e, tuttavia, tale confine in Husserl tenderà sempre ad assottigliarsi fino quasi a scomparire. (1) e (2) sono certamente analitiche in quanto riconducibili a una forma proposizionale sempre vera. Dobbiamo, però, anche notare come le forme linguistiche stesse siano, a loro volta, una varietà formale definita, cui riconduciamo oggetti materiali, e cioè gli enunciati di un linguaggio. L'operazione, dunque, non è tanto diversa da quella che compiamo riportando le case e i tetti alla struttura formale intero-parte - per rimanere nell'ambito del nostro esempio. Tra (10) e (11) non c'è lo stesso rapporto che c'è tra (1) e la sua forma proposizionale - e questo lo abbiamo già sottolineato -; ma tra (1) e la sua forma proposizionale c'è lo stesso che rapporto che sussiste tra il riferimento di (10) e il riferimento di (11). In un certo senso, dunque, anche (1) è analitica per ragioni ontologiche. Pertanto, è bene per esigenze di chiarezza e schematicità in sede espositiva tracciare una distinzione tra i due sensi linguistico e ontologico - di "analiticità" che emergono in Husserl, legandoli alla doppia articolazione della sfera del formale. Tuttavia, al contempo è altrettanto corretto ricordare che questa divisione non va estremizzata, ma intesa sempre nell'ottica di una duplicità ineliminabile propria del formale in quanto tale. 
neo-empiristi è questo: a scontrarsi non sono solo due differenti concezioni dell'analiticità, ma anche due differenti modi di intendere il significato del termine "analitico". Bisogna, perciò, evitare confusioni. Una tale accortezza non è stata usata dai protagonisti del dibattito che abbiamo ricostruito. Per quanto ci riguarda, invece, non possiamo permetterci di non segnalare e di non tenere in conto eventuali oscillazioni di significato, ogni qual volta esse si presentino, se vogliamo intendere chiaramente ciò di cui si sta parlando effettivamente e ricostruirlo. In questo senso, dovrebbe essere ormai chiaro che quanto ha in mente Husserl è la distinzione formale/materiale ${ }^{40}$, così come dovrebbe essere chiaro che quest'ultima non coincide pienamente con la distinzione analitico/sintetico proposta dai neo-empiristi.

Pertanto, se vogliamo evitare confusioni, abbiamo di fronte a noi due possibilità. La prima opzione è questa: ogni volta che parliamo di Husserl, mettere da parte la distinzione analitico/sintetico, sostituendola con quella formale/materiale. La seconda consiste nel definire prima un uso standard dei termini "analitico" e "sintetico", sulla base di (C), e introdurre successivamente un uso husserliano di quei termini. L'importante, in ogni caso, è non usare "analitico/ sintetico" nel senso standard e dire che esso coincide con "formale/materiale", pena il rischio di non comprendere ciò che Husserl aveva in mente, cadendo quindi in pericolosi equivoci.

40 Cfr. Husserl 1968, p. 44. 


\section{Riferimenti bibliografici}

Awodey 2007: Steve Awodey, Carnap's Quest for Analiticity: the Studies in Semantics in M. Friedman and R. Creath (eds) Cambridge Companion to Carnap, Cambridge University Press, Cambridge-Massachusets 2007, pp. 226-247

Benoist 1997: Jocelyn Benoist, De Kant à Bolzano: Husserl et l'analyticité, «Revue de métaphysique et de morale», No. 2, 1997, pp.217-238.

Bolzano 1973: Bernhard Bolzano, Theory of Science (Edited, with an Introduction, by Jan Berg, Translated from German by Burnham Terrell) Reidel Publishing Co., Dordrecht 1973.

Carnap 1956: Rudolf Carnap, Meaning and Necessity. A Study in Semantics and Modal Logic, The University of Chigago Press, Chicago-Illinois 1956.

Coffa 1987: Albero J. Coffa, Carnap, Tarski and the Search for Truth, «Noûs», Vol. 21, No. 4 (Dec., 1987), pp. 547572 .

Hacking 1979: Ian Hacking, What is Logic?, «The Journal of Philosophy», Vol. 76, No. 6 (Jun., 1979), pp. 285-319

Husserl 1966: Edmund Husserl, Logica formale e trascendentale. Saggio di critica della ragione logica, traduzione italiana di Guido Davide Neri, Laterza, Roma-Bari 1966.

Husserl 1968: Edmund Husserl, Ricerche logiche. Volume II, traduzione italiana di Giovanni Piana, Il Saggiatore, Milano 1968.

Husserl 2001: Edmund Husserl, Filosofia dell'aritmetica, traduzione italiana di Giovanni Leghissa, Bompiani, Milano 2001.

Husserl 2002a: Edmund Husserl, Idee per una fenomenologia pura e per una filosofia fenomenologica. Libro primo: Introduzione generale alla fenomenologia pura, traduzione italiana di Vincenzo Costa, volume I, Einaudi, Torino 2002.

Husserl 2002b: Edmund Husserl, Idee per una fenomenologia pura e per una filosofia fenomenologica. Libro secondo: Ricerche fenomenologiche sulla costituzione, traduzione italiana di Vincenzo Costa, volume II, Einaudi, Torino 2002.

Kant 2000: Immanuel Kant, Critica della ragion pura, traduzione italiana di Giovanni Gentile e Giuseppe LombardoRadice (1909/10) rivista da Vittorio Mathieu (1959), Laterza, Roma-Bari 2000 (10 edizione).

Kripke 1980: Saul Kripke, Naming and Necessity, Basil Blackwell, Oxford 1980.

Miraglia 2006: Roberto Miraglia, Dove iniziano gli a priori materiali? Schlick, Wittgenstein e le radici di un equivoco in Roberta Lanfredini (a cura di), A priori materiale. Uno studio fenomenologico, Guerini e associati, Milano 2006, pp. 95-120.

Parrini 2002: Paolo Parrini, L'empirismo logico. Aspetti storici e prospettive teoretiche, Carocci, Roma 2002.

Parrini 2006: Paolo Parrini, A priori materiale e forme trascendentali della conoscenza. Alcuni interrogativi epistemologici in Roberta Lanfredini (a cura di), A priori materiale. Uno studio fenomenologico, Guerini e associati, Milano 2006, pp. 15-39.

Proops 2009: Ian Proops, Wittgenstein's Logical Atomism, «The Stanford Encyclopedia of Philosophy» (Summer 2009 Edition), Edward N. Zalta (Ed.), URL= http://plato.stanford.edu/entries/wittgenstein-atomism.

Putnam 1975: Hilary Putnam, "The Analytic and the Synthetic" in Hilary Putnam, Mind, Language and Reality. Philosphical Papers. Volume 2, Cambridge University Press, Cambridge-Massachusets 1975, pp. 33-69.

Quine 1961: Willard Van Orman Quine, Two Dogmas of Empiricism in Willard Van Orman Quine, From a Logical Point of View. Nine Logico-Philosophical Essays, Harvard University Press, Cambridge-Massachusets 1961, pp. 20-46. 
Quine 1966: Willard Van Orman Quine, Carnap and Logical Truth in Willard Van Orman Quine, The Ways of Paradox and Other Essays, Random House, New York 1966, pp. 101-125.

Ramsey, Moore 1927: Frank Plumpton Ramsey, George Edward Moore, Facts and Propositions, «Proceedings of the Aristotelian Society» Supplementary Volumes, Vol. 7, Mind, Objectivity and Fact (1927), pp. 153-206.

Schlick 1987a: Moritz Schlick, Esiste un a priori materiale? In Moritz Schlick, Forma e contenuto, traduzione italiana di Paolo Parrini, Bollati Boringhieri, Torino 1987, pp. 167-179.

Schlick 1987b: Moritz Schlick, Foma e contenuto. Un'introduzione al pensare filosofico in Moritz Schlick, Forma e contenuto, traduzione italiana di P. Parrini, Bollati Boringhieri, Torino 1987, pp. 45-148.

Sher 1991: Gila Sher, The Bounds of Logic. A Generalized Point of View, The MIT Press, Cambidge-Massachusets 1991.

Simons 1992: Peter Simons, Philosophy and Logic in Central Europe from Bolzano to Tarski. Selected Essays, Kluwer, Dordrecht 1992.

Smith, Murray 1981: Barry Smith, David Murray, Logic, Form and Matter, «Proceedings of the Aristotelian Society» Supplementary Volumes, Vol. 55 (1981), pp. 47-63+65-74.

Spinicci 2007: Paolo Spinicci, Analitico e sintetico. Lezioni su Kant, Husserl, Quine, CUEM, Milano 2007.

Tarski 1956: Alfred Tarski, Logic, Semantics, Metamathematics. Papers from 1923 to 1938, Hackett Pusling Company, Oxford 1956.

Waismann 1975: Friederich Waismann, Ludwig Wittgenstein e il Circolo di Vienna. Colloqui annotati da Friederich Waismann, traduzione italiana Sabina de Waal, La Nuova Italia, Firenze 1975.

Wittgenstein 1929: Ludwig Wittgenstein, Some Remarks on Logical Form, «Proceedings of the Aristotelian Society», Supplementary Volumes, Vol. 9 (1929), pp. 162-171. 